\title{
Making Planets on Earth: \\ How Experimental Petrology Is Essential to Planetary Exploration
}

\section{Primary Author:}

Kayla Iacovino ${ }^{1, *}$

\section{Co-authors:}

Nicole G. Lunning 2 , Gordon M. Moore ${ }^{1}$, Kathleen Vander Kaaden ${ }^{1}$, Kevin Righter ${ }^{2}$, Francis M. McCubbin $^{2}$, Kelsey B. Prissel ${ }^{3}$, Paul D. Asimow ${ }^{4}$

\section{Co-signers:}

Benjamin J. Andrews ${ }^{5}$, Don R. Baker ${ }^{6}$, J. Brian Balta ${ }^{7}$, Jeremy Boyce ${ }^{2}$, Karalee K. Brugman ${ }^{3}$, Eric H. Christiansen ${ }^{8}$, Max Collinet ${ }^{9}$, Elizabeth Cottrell ${ }^{5}$, David Dobson ${ }^{10}$, David S. Draper ${ }^{11}$, Nicholas Dygert $^{12}$, Stephen M. Elardo ${ }^{13}$, Katy A. Evans ${ }^{14}$, Patrizia Fumagalli ${ }^{15}$, Julia Hammer ${ }^{16}$, Daniel Harlov $^{17}$, Marc M. Hirschmann ${ }^{18}$, Astrid Holzheid ${ }^{19}$, Stephan Klemme ${ }^{20}$, Kurt Leinenweber ${ }^{21}$, Simon Matthews ${ }^{22}$, Monika Koch-Müller ${ }^{17}$, Stephan Kolzenburg ${ }^{23}$, Andrea Koziol ${ }^{24}$, Michael J. Krawczynski ${ }^{25}$, Charles E. Lesher ${ }^{26}$, Jie (Jackie) $\mathrm{Li}^{27}$, Craig Manning ${ }^{28}$, Timmothy $\mathrm{McCoy}^{5}$, Celestine Mercer $^{29}$, Tabb C. Prissel ${ }^{30}$, Xiaofei $\mathrm{Pu}^{31}$, Geneviève Robert ${ }^{32}$, Christopher A. Rochelle ${ }^{33}$, Tracy Rushmer ${ }^{34}$, Christy B. Till ${ }^{21}$, Jack P. Touran ${ }^{1}$, Dustin Trail ${ }^{35}$, Allan H. Treiman $^{30}$, James Van Orman ${ }^{36}$, Wim van Westrenen ${ }^{37}$, Alan Whittington ${ }^{38}$, Penny E. Wieser ${ }^{39}$, Max Wilke ${ }^{40}$, Youxue Zhang ${ }^{27}$

*Phone: 832-413-1908, email: kayla.iacovino@nasa.gov

1 Jacobs, NASA Johnson Space Center, Houston, TX, USA

2 NASA Johnson Space Center, Houston, TX, USA

${ }^{3}$ Earth and Planets Laboratory, Carnegie Institution for Science, Washington DC, USA

${ }^{4}$ California Institute of Technology, Pasadena, CA, USA

${ }^{5}$ National Museum of Natural History, Smithsonian, Washington DC, USA

${ }^{6}$ McGill University, Montreal, Canada

7 Texas A\&M University, College Station, TX, USA

${ }^{8}$ Brigham Young University, Provo, UT, USA

${ }^{9}$ Institute of Planetary Research, German Aerospace Center (DLR), Germany

${ }^{10}$ University College London, London, UK

${ }^{11}$ NASA Headquarters, Washington DC, USA

${ }^{12}$ University of Tennessee, Knoxville, TN, USA

${ }^{13}$ University of Florida, Gainesville, FL, USA

${ }^{14}$ Curtin University, Australia

${ }^{15}$ Universita degli Studi di Milano, Italy

${ }^{16}$ University of Hawaii Manoa, Honolulu, HI, USA

${ }^{17}$ Helmholtz-Zentrum Potsdam, Germany

${ }^{18}$ University of Minnesota, Minneapolis, MN, USA

${ }^{19}$ Universität Kiel, Kiel, Germany

${ }^{20}$ University of Munster, Munster, Germany

${ }^{21}$ Arizona State University, Tempe, AZ USA

${ }^{22}$ Johns Hopkins University, Baltimore, MD, USA

${ }^{23}$ University at Buffalo, NY, USA

${ }^{24}$ University of Dayton, OH, USA

${ }^{25}$ Washington University in St. Louis, Missouri, U.S.A

${ }^{26}$ University of California Davis, CA, USA

${ }^{27}$ University of Michigan, Ann Arbor, MI, USA

${ }^{28}$ University of California Los Angeles, CA, USA

${ }^{29}$ US Geological Survey, Denver, CO, USA

${ }^{30}$ Lunar and Planetary Science Institute, USRA, Houston, TX, USA

${ }^{31}$ Idaho National Laboratory, Idaho Falls, ID, USA

${ }^{32}$ Bates College, Lewiston, ME, USA

${ }^{33}$ British Geological Survey, Keyworth, Nottingham, UK

${ }^{34}$ Macquarie University, Australia

${ }^{35}$ University of Rochester, NY, USA

${ }^{36}$ Case Western University, Cleveland, OH, USA

${ }^{37}$ Vrije Universiteit Amsterdam

${ }^{38}$ University of Texas at San Antonio, TX, USA

${ }^{39}$ University of Cambridge, UK

${ }^{40}$ Universitat Potsdam, Germany 
1.0 Introduction Our knowledge of the evolution of the solar system relies upon our ability to interpret a limited set of observations, including geochemical data from returned samples and meteorites, remotely sensed data from spacecraft and telescopes, and information measured in situ via landers and rovers. Our ability to obtain these data is constantly improving in quality and quantity, but the understanding of the universe derived from these data is only as good as our interpretive tools. Experimental petrology is the most powerful way in which we define fundamental geochemical principles, e.g., thermodynamic equations of state, volatile solubilities and partitioning, partitioning of elements between gaseous, fluid, silicate and/or metal phases, and pressure-temperature-redox-composition-time controls on phase composition, stability, and texture. These laboratory-derived insights are critical to precisely translating hard-won geochemical data sets into constrained models of the formation and evolution of our solar system.

Experimental petrology is a laboratory approach where the conditions within planetary bodies - from atmospheres to cores - are reproduced and measured in a controlled environment. In this way, it is possible to investigate the precise pressure $(P)$, temperature $(T)$, redox $\left(f \mathrm{O}_{2}\right)$, compositional $(X)$, and in some cases time $(t)$ conditions under which natural rocks form. Experimental petrology provides three fundamental services. First, the intensive parameters $(P$, $\left.T, f O_{2}, X\right)$ of specific rocks may be determined by "reverse engineering" the conditions that match characteristics such as mineral assemblage, element concentration and partitioning, etc. This can be useful for understanding the evolution of a rock or suite of rocks and how they relate to the planetary body as a whole. Second, broader strategic experimental campaigns can map swaths of parameter space (e.g., $P-T, T-t, X$ - $t$ ) systematically. This, in turn, provides specific insight into fundamental relationships among geochemical variables in specific meteorite or rock systems and, over time, builds a database for calibrating thermodynamic or empirical models capable of predicting rock formation conditions. Third, non-equilibrium measurements of kinetic processes such as crystallization, exsolution, and vesiculation; and quantification of diffusivities, elasticities, viscosities and more are important contributions that experimental

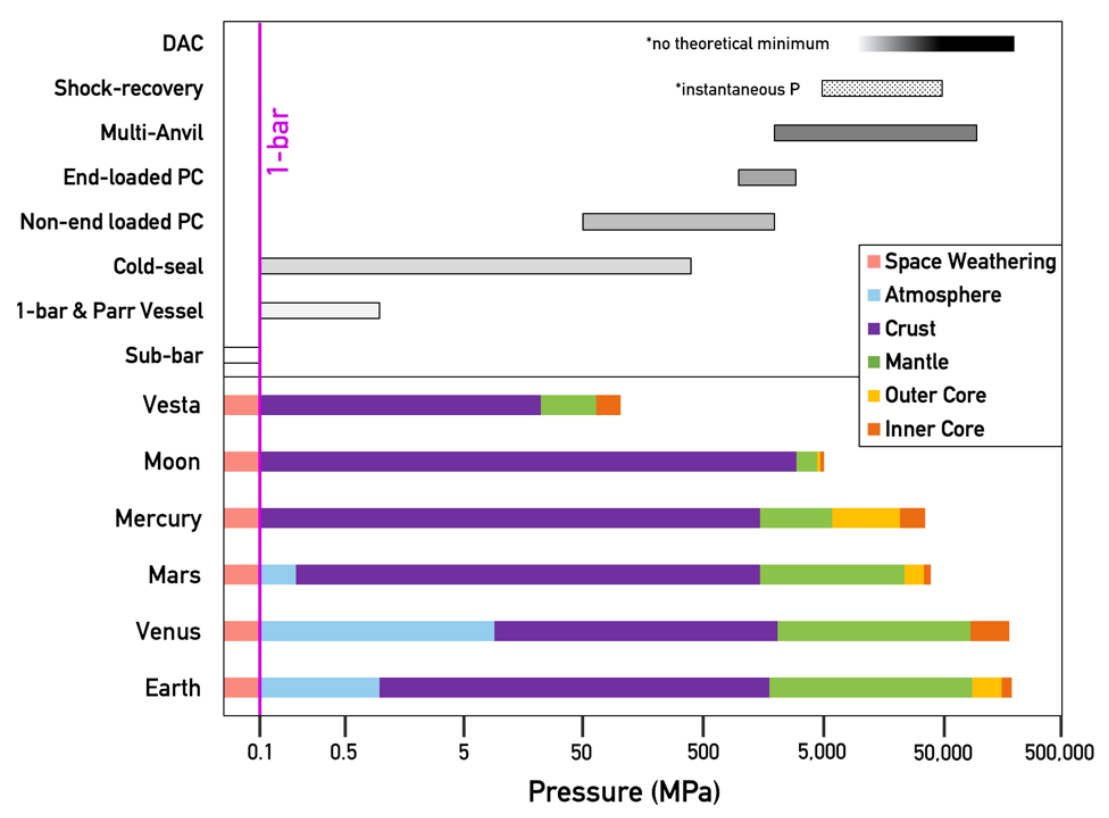

Figure 1. Pressures achieved with experimental apparatus described here (top) and corresponding pressures of planetary layers within some solar system bodies (bottom). X-axis is log scale. petrologists can make to solve problems in planetary science. These approaches develop our understanding of the fundamental processes that built and modified our solar system over the last 4.5 billion years. In the following sections, we detail experimental methodologies employed to investigate each major geologic regime in planetary bodies (Figure 1). Examples where experimental petrology has formed the basis of major advancements in our understanding of the universe are given, exemplifying the ongoing need for petrologic 
experimentation to be a continuously funded line of planetary science research in the decade to come. In each section, we highlight gaps in our knowledge and outline experimental work needed to meet new research goals. In addition to the continued funding of experimental petrology over the next decade, funds must also be available to develop new experimental apparatuses and techniques that are directly in line with the planned future missions.

2.1 Planetary atmospheres and surfaces Planetary atmospheres can range from relatively inert (e.g., contemporary Mars) to relatively reactive (e.g., Venus), with radically differing consequences for the chemical rocks at planetary surfaces. The compositions of atmosphere and fluids have important implications for habitability conditions on Europa, Titan, and ancient Mars. As we learn more about current and past atmospheres of these bodies over the next decade, it will be possible to run targeted laboratory experiments to expand our understanding of the geochemical processes at work. In addition, atmosphere-surface fluid, rock-atmosphere, ice-atmosphere, and fluid-rock interactions can be geochemically preserved in surface rock deposits from which complementary experimental work can be used to elucidate past atmosphere and/or surface fluid compositions. Crucially, the uppermost rocky layer is the only portion of planetary bodies routinely measured via remote sensing and even rover and lander operations. As such, understanding the reactions taking place in planetary atmospheres has a direct bearing on the interpretation of the principal geochemical observations we have of other bodies.

Aqueous alteration processes played an important role on some carbonaceous chondrite parent bodies and modified the organic building blocks for life found in some meteorites ${ }^{1,2}$. We anticipate learning more about these organic building blocks from the samples that will be returned by OSIRIS-REx and Hayabusa2. In addition, improvements in our telescopic observation capabilities (e.g., the James Webb Space Telescope) may strengthen connections between carbonaceous chondrite meteorite groups and specific asteroids, which would allow for estimation of parent body sizes and potential pressure constraints for early solar system aqueous alteration processes. The potential discoveries above will warrant new experimental techniques to understand the conditions needed to preserve specific suites of organic components and to advance our understanding of processes related to water in the early solar system.

Planetary atmospheres from near-vacuum up to several bars can be recreated with an array of instruments. The most common method is the reproduction of atmospheres at Earth-ambient pressure in 1-bar gas-mixing furnaces. Such experiments have been commonplace for decades and, because thermodynamic properties of gases are well known at 1 bar, form the empirical backbone of datasets extrapolated to higher or lower pressures. At low P-T conditions, analytical instruments can themselves serve as experimental apparatus. Cryogenic electron microscopy (cryo-SEM, cryo-TEM) techniques have been used to characterize both natural and lab-grown ice and gas hydrates formed under 1 bar or greater pressure conditions ${ }^{3}$. Higher pressure atmospheres up to several bars can be simulated in closed reaction "Parr" vessels, in which reagents combined and sealed in a constant volume chamber are heated. Elevated pressure results from the thermal expansion of reagents. These pressures are higher than the surface pressure on Mars, Mercury, and the exteriors of planetesimals. Some of the reactions occurring on these bodies are likely to be very pressure sensitive, and so to fully understand these worlds, we need to conduct experiments at the relevant pressures, below that of the ambient Earth. Vacuum furnaces and evacuated, sealed silicaglass tubes have been used to understand nebular condensation and degassing processes, including stable isotope fractionation, but these apparatuses have primarily been used to study the residual melt and infer the products that were lost via mass balance. Recent advances in the experimental 
community have enabled the development of sub-bar experimental systems designed to capture the residual melt, condensed solids that form from the degassed vapor, and any uncondensed gas. This "sulb-bar furnace" consists of a vacuum system connected to an unpressurized tube furnace. Glass vacuum lines are run directly from the sample into a gas chromatograph, such that measurements of outgassing can be measured in situ at high temperatures or captured within a cold trap for subsequent analysis. Experiments from this system will enable our community to explore completely new questions about the gas and/or fluid compositions that exsolve from rocks or lavas on planetary bodies that have atmospheric pressures below 1 bar, such as Mercury, Mars, and differentiated asteroids.

2.2 Planetary crusts and small bodies Experiments performed at crustal conditions have enabled countless geochemical models for interpreting solar system bodies. These studies have firmly established that petrologic reservoirs are unique to certain planetary bodies, and that redox conditions vary between different bodies. Studies of melt solubilities and crystal-melt partitioning of volatiles $(\mathrm{H}, \mathrm{C}, \mathrm{S}, \mathrm{F}, \mathrm{Cl})$ support determination of volatile contents in the interiors of other planets, the Moon, and asteroid parent bodies ${ }^{4-6}$. These studies ultimately help us to build a picture of the sources of volatiles in the solar system at large. Experimental studies have been critical in shaping our understanding of density-driven planetary crust formation and were crucial in forming the current theories for the formation of the lunar highlands ${ }^{7}$ and mare ${ }^{8}$.

The crusts of rocky bodies dramatically vary in their geochemical compositions across the solar system. The extremes of this crustal compositional range are arguably the Moon's feldspathic highlands, Earth's granitoid continental shields, and Mercury's MgO-rich and FeO-poor boninitic crust, as recently revealed by the MESSENGER mission ${ }^{9,10}$. Extreme crusts like that of Mercury have no terrestrial analog and indeed our collections contain no Mercurian meteorites. Thus, experimental petrology is the primary way we investigate the composition ${ }^{9,10}$ and rheology ${ }^{11}$ of Mercury. Laboratory spectral analysis of synthetic analog materials can also provide "ground truth" for remotely sensed spectral observations of unique and potentially unsampled lithologies. In this way, synthesis experiments at controlled conditions aid in interpreting mineral chemistry and abundance from remote measurements of planetary surfaces, such as those from the Moon Mineralogy Mapper on Chandrayaan-1 or Diviner aboard the Lunar Reconnaissance Orbiter.

Crusts with basaltic compositions are common; Venus, Mars, and asteroid 4 Vesta have mostly basaltic crusts, as do the lunar mare and terrestrial oceanic crust ${ }^{12}$. Terrestrial experiments can be helpful in interpreting our observations of these bodies, but distinct differences in conditions on these worlds (e.g., gravitational acceleration, atmospheric pressure, etc.) mean that specialized experimentation is required beyond what is available in the terrestrial literature. In addition, studies of disequilibrium processes becoming more commonplace in the terrestrial community should also be leveraged in planetary science in the coming decade. Investigations of volcanic eruption triggering via gas overpressure ${ }^{13}$ could elucidate the conditions for lunar fire fountaining, and the origins of chondrules could be constrained based on experimentally reproducing their textures ${ }^{14,15}$.

Experimental studies of planetary crusts have largely been undertaken in either (a) 1-bar gas-mixing furnaces $(0.1 \mathrm{MPa})$ or (b) piston cylinder apparatuses at pressures of 500-3,000 $\mathrm{MPa}$, simulating surface to upper-mantle depths (Figure 1). Experiments at pressures which correspond to intermediate depths between the surface and upper mantle could substantially advance our understanding of volatile retention/degassing, as well as potential corresponding magmatic signatures. Experiments at these intermediate pressures (0.1-400 MPa) are accessible with cold-seal experimental apparatuses and are widely used by the terrestrial experimental 
petrology community but have only been used in few studies of extraterrestrial petrology. Recent experimental approaches have sought novel ways to study the magmatic processes at intermediate depths between the surface and upper mantle, particularly to investigate pressure sensitive components such as volatiles that could influence atmospheric composition of their planetary body or fluid compositions that may alter shallow crustal environments ${ }^{16}$. These include experiments in 1-bar furnaces that capture chemical species that are lost from the experimental magmas due to volatilization ${ }^{16}$.

The pressure range for the crustal depths of approximately Earth-sized planets $(0.1-1,000$ $\mathrm{MPa}$ ) also encompasses the full range of pressures within interiors of small solar system bodies (Figure 1), such as planetesimals and their contemporary descendants in the asteroid belt. Experimental petrology is particularly important for understanding planetesimals because our ability to observe most of these bodies is limited to telescopic observations, exploration by only a handful of spacecraft (although there will be more in the next decade), and incomplete sampling of these bodies as in-falling meteorites. For example, we have iron meteorites that represent the cores of at least 50 separate differentiated planetesimals but we do not have crustal or mantle samples from that many bodies in our meteorite collections. Experimental studies continue to help us to explain the processes that planetesimals underwent in the early solar system and to identify and determine the properties of the components missing from our sample collections.

In the coming decade, the Psyche Mission will explore the metallic asteroid 16 Psyche that is thought to be the core of a differentiated planetesimal. The Psyche mission is anticipated to reveal the composition of the outer core of its planetesimal, and some recent astronomical observations suggest core-mantle boundary regions may be exposed on the surface of this metallic asteroid. These anticipated observations will provide humanity's first look at the interior structures of differentiated planetesimals, which will be a jumping off point for experiments aimed at understanding the differentiation processes that formed Psyche and how planetesimal core solidification proceeded (e.g., some models include inward core solidification) ${ }^{17}$. The model for differentiation and core solidification for Psyche will then be compared with additional meteorites and new experiments to understand the diversity (or lack thereof) in differentiation and core solidification processes in the early solar system.

2.3 Planetary mantles As a limited number of direct samples from the (upper terrestrial) mantle are available, petrologic experiments provide geochemical information to complement and interpret what we know about Earth's interior structure (mantle and core) from geophysical measurements. With experiments, we can use the most primitive (mantle-derived) available basaltic compositions to reverse engineer the type of mantle material that would have undergone partial melting to form that specific type of basalt ${ }^{18}$. For example, mantle compositions estimated from Martian meteorite compositions indicate there are two geochemically distinct mantle reservoirs, but there remain many open questions about what these different mantle reservoirs indicate about the martian interior ${ }^{5}$. In addition, geochemical information about planetary mantles can be used to constrain rheology; where are mantle melts generated? Are they more or less buoyant than surrounding solid mantle ${ }^{19}$ ? What processes drive differentiation in late-stage magma oceans, and what are the physical and geochemical controls on these processes ${ }^{20}$ ?

Experiments on planetary mantles are typically carried out in solid-media pressure apparatuses capable of generating the relevant high pressures (piston cylinders (PCs) and multianvils). These greatly differ from gas or fluid pressurized apparatuses used at low pressures in that samples are enclosed within a multi-component (typically ceramic) assembly and pressurized by 
applying force in one or multiple directions using hydraulic presses. The extreme pressures and temperatures found in planetary mantles make these experiments extremely difficult to perform, with high failure rates and steep thermal gradients across samples. Despite these challenges, highpressure experimentation remains one of the foremost ways in which we constrain deep planetary processes, from differentiation and core formation to the interpretation of geophysical data.

2.4 Planetary cores In bodies large enough to undergo differentiation into a metallic core and silicate mantle and crust, understanding the nature and rate of elemental and isotopic equilibration between metal and silicate is critical for interpreting chemical, chronologic, and genetic data. Metal-silicate partitioning for several key elements (typically siderophile elements such as W, Mo, $\mathrm{Ru}, \mathrm{Cr}, \mathrm{Sn}, \mathrm{Pt}$, etc.) has been studied extensively using experimental techniques ${ }^{21-23}$. Other studies have led to a better understanding of the composition of Earth's core, including what concentration(s) of light element (H, S, Si, etc.) could explain geophysical data ${ }^{24}$. To constrain partitioning over the wide $P-T$ space relevant to planetesimals and planets, experiments of this type may utilize a variety of the experimental techniques described in this paper, from 1 bar $(0.1 \mathrm{MPa})$ up to thousands of MPa, with higher pressures obtained using multi-anvil presses. Extremely high pressures replicating conditions in the cores of Earth-sized bodies can be obtained with diamondanvil cells (DACs), which house a very small sample pressurized between two precisely machined diamonds.

Of particular interest near term is in constraining the partitioning behavior and equilibration timescales related to isotopic equilibrium of siderophile elements between silicate and metal. Currently isotopic experimental data are incomplete or absent. As a result, modeling the extent of equilibrium during accretion modeling of inner solar system bodies varies from full isotopic equilibrium to very limited equilibrium. For example, isotopic heterogeneities observed in the terrestrial mantle and derivative samples (basalts, komatiites) could be attributed to the addition of late veneer material to the Earth after core formation, or they could reflect a lack of isotopic equilibrium over the timeframe of mantle evolution ${ }^{20}$. Isotopic data in planetary materials have been measured for a wide range of siderophile elements including $\mathrm{Mo}, \mathrm{W}, \mathrm{Ru}, \mathrm{Cr}, \mathrm{Sn}, \mathrm{Pt}, \mathrm{Zn}, \mathrm{Cu}$, $\mathrm{V}$, Os, S, and Si. Without the corresponding thermodynamic information (i.e., partition coefficients and equilibration timescales) however, interpreting these data is difficult. In addition, disequilibrium studies into, for example, diffusion rates in metal-bearing systems could be used to infer parent body dimensions and cooling rates from diffusion profiles or metallographic textures in iron meteorites ${ }^{25}$. In order to fully leverage our existing and continually growing datasets, we suggest that future experimental studies of metal-silicate systems should target the isotopic partitioning and disequilibrium processes of these critical elements.

2.5 Planetary impacts Hypervelocity impact is one of the most significant processes in shaping planetary surfaces, modifying meteorite parent bodies, and-especially in early solar system history-determining the large-scale architecture of planets and satellites. The small-scale evidence of impact history-damage, fracture, amorphization, recrystallization, unique highpressure mineral assemblages, and melt veins - can all be reproduced experimentally via dynamic "shock recovery" experiments, which produce instantaneous pressures from 5,000-50,000 MPa (in contrast to sustained high-pressure experiments). Typically, samples are enclosed in steel chambers and subjected to gun-driven plate impacts to propagate shock waves through chamber and sample; the sample can then be recovered intact and studied microscopically in much the same way as quenched samples from static experiments ${ }^{26}$. Threshold pressures for dynamic phase 
transitions can then be compared to equilibrium phase diagrams constructed from static experiments to refine estimates of the impact conditions recorded by shocked samples from meteorites and planetary surfaces ${ }^{27}$. Frontier research areas in shock recovery include alternative loading pathways that reach higher or lower temperatures than traditional reverberating shocks, use of new high-resolution analytical tools to probe smaller scales, and laser-driven loading platforms.

3.0 Concluding remarks The success of missions like BepiColombo, Mars 2020, MMX, OSIRISREx, Hayabusa2, and Psyche over the next decade will provide us with unprecedented geochemical and petrologic data of solid planetary bodies across our solar system. In order to not only prepare for the interpretations of these complex datasets, but to enhance the scientific return of this data from these various missions, a wide range of experimental petrology studies are warranted; a select few were detailed in this paper. In addition to experimental equipment, microanalytical instrumentation (e.g., electron, ion, and laser beam analyses, vibrational spectroscopy, and synchrotron-based methods) is crucial for analyzing both natural and experimentally produced samples. Petrologic experimentation and analysis provide a ground-truth empirical and thermodynamic foundation on which to build interpretations of these various datasets. Funding should be allocated toward expanding current capabilities into new crucial areas of research including, for example, high $\mathrm{P}$ low $\mathrm{T}$ equipment to study exotic ices; in situ measurements at high and low P-T; and the simulation of disequilibrium processes. To attain these goals, it is imperative that the decadal survey consider the role of equity, diversity, inclusion, and accessibility in planetary science. To truly understand the thermochemical and petrologic evolution of rocky bodies across our solar system, it is imperative that experimental petrology remain an active and diverse line of research in the coming decade.

1. Lipschutz, M. E. \& Schultz, L. Meteorites in Encyclopedia of the Solar System (Second Edition), Chapter 13. (2007).

2. Brearley \& A.J. The Action of Water. Meteorites and the early solar system I/ 943, (2006).

3. Blake, D., Allamandola, L., Sandford, S., Hudgins, D. \& Freund, F. Clathrate hydrate formation in amorphous cometary ice analogs in vacuo. Science 254, 548-551 (1991).

4. McCubbin, F. M. et al. Experimental investigation of $\mathrm{F}, \mathrm{Cl}$, and $\mathrm{OH}$ partitioning between apatite and Fe-rich basaltic melt at 1.0$1.2 \mathrm{GPa}$ and $950-1000{ }^{\circ} \mathrm{C}$. Am Mineral 100, 1790-1802 (2015).

5. Barnes, J. J. et al. Multiple early-formed water reservoirs in the interior of Mars. Nat Geosci 13, 260-264 (2020).
6. Dasgupta, R., Chi, H., Shimizu, N., Buono, A. S. \& Walker, D. Carbon solution and partitioning between metallic and silicate melts in a shallow magma ocean: Implications for the origin and distribution of terrestrial carbon. Geochim Cosmochim Ac 102, 191-212 (2013).

7. Walker, D. \& Hays, J. F. Plagioclase flotation and lunar crust formation. Geology 5, 425 (1977).

8. Smith, J. V., Anderson, A. T., Newton, R. C., Olsen, E. J. \& Wyllie, P. J. A Petrologic Model for the Moon Based on Petrogenesis, Experimental Petrology, and Physical Properties. J Geology 78, 381-405 (1970).

9. Kaaden, K. E. V. \& McCubbin, F. M. The origin of boninites on Mercury: An experimental study of the northern volcanic plains lavas. Geochim Cosmochim Ac 173, 246-263 (2016). 
10. Kaaden, K. E. V. \& McCubbin, F. M. Exotic crust formation on Mercury: Consequences of a shallow, FeO-poor mantle. J Geophys Res Planets 120, 195-209 (2015).

11. Sehlke, A. \& Whittington, A. G. Rheology of lava flows on Mercury: An analog experimental study: MERCURY LAVA RHEOLOGY. J Geophys Res Planets 120, 1924-1955 (2015).

12. Condie \& K.C. Comparative Planetary Evolution in Earth as an Evolving Planetary System (Third Edition), Chapter 10. in 317-367 (n.d.).

13. Arzilli, F. et al. Magma fragmentation in highly explosive basaltic eruptions induced by rapid crystallization. Nat Geosci 12, 1023-1028 (2019).

14. Desch, S. J., Morris, M. A., Connolly, H. C. \& Boss, A. P. The importance of experiments: Constraints on chondrule formation models. Meteorit Planet Sci 47, 1139-1156 (2012).

15. Jones, R. H., Villeneuve, J. \& Libourel, G. Thermal histories of chondrules: Petrologic observations and experimental constraints. Chondrules and Protoplanetary Disk 57-90 (2017) doi:10.1017/9781108284073.003.

16. Ustunisik, G., Nekvasil, H. \& Lindsley, D. Differential degassing of $\mathrm{H} 2 \mathrm{O}, \mathrm{Cl}, \mathrm{F}$, and $\mathrm{S}$ : Potential effects on lunar apatite. Am Mineral 96, 1650-1653 (2011).

17. Scheinberg, A., Elkins-Tanton, L. T., Schubert, G. \& Bercovici, D. Core solidification and dynamo evolution in a mantle-stripped planetesimal. $J$ Geophys Res Planets 121, 2-20 (2016).

18. Till, C. B. A review and update of mantle thermobarometry for primitive arc magmas. Am Mineral 102, 931-947 (2017).
19. Fu, R. R. \& Elkins-Tanton, L. T. The fate of magmas in planetesimals and the retention of primitive chondritic crusts. Earth Planet Sc Lett 390, 128-137 (2014).

20. Trønnes, R. G. et al. Core formation, mantle differentiation and core-mantle interaction within Earth and the terrestrial planets.

Tectonophysics 760, 165-198 (2019).

21. Righter, K. \& Shearer, C. K. Magmatic fractionation of $\mathrm{Hf}$ and $\mathrm{W}$ : constraints on the timing of core formation and differentiation in the Moon and Mars. Geochim Cosmochim Ac 67, 2497-2507 (2003).

22. Touboul, M., Walker, D., Ash, R. D., Puchtel, I. S. \& Walker, R. J. Simultaneous experimental determination of metal-silicate partitioning of $\mathrm{W}$, $\mathrm{Mo}, \mathrm{Ru}, \mathrm{Pt}$, and $\mathrm{Pd}$ using natural abundances, elevated P-T and isotopic tracers. Lunar and Planetary Science Conference 42, 1727 (2011).

23. Wade, J., Wood, B. J. \& Tuff, J. Metal-silicate partitioning of $\mathrm{Mo}$ and $\mathrm{W}$ at high pressures and temperatures: Evidence for late accretion of sulphur to the Earth. Geochim Cosmochim Ac 85, 58-74 (2012).

24. McDonough, W. F. Compositional Model fo the Earth's Core. in 559-576 (2003).

25. Watson, H. C., Richter, F., Liu, A. \& Huss, G. R. Iron and nickel isotope fractionation by diffusion, with applications to iron meteorites. Earth Planet Sc Lett 451, 159-167 (2016).

26. Elamin, M. \& Varga, J. Plate impact method for shock physics testing. Mat Sci \& Eng Int/ J 4(1), 31-35 (2020).

27. Stöffler, D., Hamann, C. \& Metzler, K. Shock metamorphism of planetary silicate rocks and sediments: Proposal for an updated classification system. Meteorit Planet Sci 53, 5-49 (2017). 